Research Article

\title{
Study on the Structure and Performance of an Antagonistic Pneumatic Bidirectional Rotary Joint
}

\author{
Hongbo Liu $\mathbb{D},{ }^{1,2}$ Guodong Sun $\mathbb{D}^{2},{ }^{2}$ Dexu Geng, ${ }^{2}$ and Junye Li $\mathbb{D}^{1}$ \\ ${ }^{1}$ Ministry of Education Key Laboratory for Cross-Scale Micro and Nano Manufacturing, \\ Changchun University of Science and Technology, Changchun 130022, China \\ ${ }^{2}$ Engineering Training Center, Beihua University, Jilin 132021, China \\ Correspondence should be addressed to Junye Li; ljy@cust.edu.cn
}

Received 7 September 2021; Revised 26 October 2021; Accepted 27 October 2021; Published 20 November 2021

Academic Editor: Le Anh Tuan

Copyright (c) 2021 Hongbo Liu et al. This is an open access article distributed under the Creative Commons Attribution License, which permits unrestricted use, distribution, and reproduction in any medium, provided the original work is properly cited.

\begin{abstract}
An antagonistic pneumatic bidirectional rotary flexible joint was developed to improve both safety and environmental adaptability of service robots and associated human interactions. The joint comprises two semicircular rotary actuators with positive and negative symmetrical distributions and a pneumatic brake. As such, it achieves forward and reverse rotations, and its damping and braking are adjustable in real time, enabling it to maintain its position. According to the force/torque balance at the free end of the rotary actuator, the rotation angle static model was established. The relationship between the actuator rotation angle, driving torque, impedance torque, and air pressure was obtained experimentally. The brake airbag was manufactured using additive manufacturing and silicone gel casting technologies. The mathematical model of the braking torque was established next, and the model was verified through experiments. Furthermore, an experimental system was constructed to carry out the air pressure-angle, air pressure-torque, and speed response experiments without the load on the joint. The results have shown that the joint can achieve any position within $\pm 68.5^{\circ}$ when the driving air pressure varies from 0 to $0.30 \mathrm{MPa}$; the time required to reach the maximum angle was $0.85 \mathrm{~s}$. The joint has shown good adjustable damping characteristics. Lastly, the braking torque reached $4.21 \mathrm{Nm}$ at $0.32 \mathrm{MPa}$, effectively maintaining the position.
\end{abstract}

\section{Introduction}

With the growth in demand for service robots, the security of human-machine interaction is becoming increasingly important [1]. As critical executive service robot components, structural characteristics, motion characteristics, and robotic arm driving ability determine both function and application levels of the service robot [2]. The traditional rotary robotic arm motion is mainly achieved through various motors, hydraulic motors, and rotary cylinders. Although it has some advantages such as high accuracy, large driving torque, and fast execution speed, most of the robotic arms lack comprehensive flexibility and have poor adaptability to unstructured environments. This seriously restricts the application and development of service robots [3]. On the contrary, the pneumatic artificial muscle has a simple structure; it is lightweight and has strong flexibility. If used as a driving element in the rotary joints of robotic arms, it can further improve the safety and environmental adaptability of the human-machine interaction $[4,5]$. Hence, studying rotary joints driven by pneumatic artificial muscles will have both theoretical and practical significance.

Currently, pneumatic rotary joints usually achieve the rotary motion through antagonistic bidirectional artificial muscles $[6,7]$ or rotary actuators [8]. For example, Fras et al. developed a pneumatic rotary actuator made of silicone rubber and reinforced with polyester fiber. The maximum actuator rotation angle was $90^{\circ}$; however, it could only rotate in one direction [9]. Furthermore, a team from the Covenant University of Nigeria developed a pneumatic soft rotary actuator to be used for gesture control. The actuator was manufactured using the molding process, and a pair of antagonistic rotary actuators was used to develop a joint enabling forward and reverse rotations [10]. The joint could 
rotate from $-16^{\circ}$ to $90^{\circ}$ by coordinating the forward and reverse actuators. However, the joint did not have a braking function, which prevented it from maintaining its position. Ainla et al. developed a flexible pneumatic actuator, which consisted of multiple chambers arranged in a circle around the central rod [11]. By driving the chambers in sequence, the central rod produces approximately square circular motion. Yan et al. developed a pure torsion flexible actuator for soft robots $[12,13]$. The outer side of the actuator includes a limiting layer to limit its axial elongation. When the spiral cavity inside the actuator is pressurized, the torsional motion will be generated. Although the design of rotary joints above fully applies the characteristics of elastic (soft) materials to achieve the rotary function, the joint stiffness cannot be adjusted actively. Additionally, the position cannot be maintained when the external load changes and can easily crack when in contact with the sharp surface [14]. In practical applications, pneumatic rotary joints should not only ensure motion flexibility but also have stable positioning and load-bearing capacity in a specific environment. Therefore, real-time changing of the rotary joint rigidity becomes an effective solution strategy.

A lot of research studies were carried out on flexible joints with variable stiffness $[15,16]$. Primarily used mechanisms and principles can be divided into the following four categories: antagonistic mechanisms [17], blocking mechanisms [18-20], electric/magnetorheological principles [21], and material phase transformation principles [22, 23]. Among them, the blocking mechanism utilizes the tiny particles filled in the inner cavity of the closed membrane. By applying the negative pressure, the relative shear stress between particles in the internal cavity increases, achieving variable structure stiffness. However, due to the randomness of the tiny particle rearrangement, it may cause a decrease in variable stiffness, reducing its repeated positioning accuracy [24]. Furthermore, the variable stiffness mechanism based on the electro/magnetorheological principle requires an additional electric or magnetic field, which increases the system complexity. The phase change principle is mainly used to achieve variable stiffness through the transformation of the aggregate state; however, the stiffness adjustment speed is rather low. Compared to other stiffness adjustment methods, the variable stiffness based on the antagonism principle has several advantages, including the simple structure and low cost, while requiring no external energy field; therefore, it has good application prospects.

Giannaccini et al. [25] developed a novel variable stiffness soft arm, which consisted of elongated and contractile pneumatic muscles; the arm stiffness adjustment range was limited. Furthermore, when two equal-length muscles worked simultaneously, mutual impedance occurred, affecting the muscle deformations. Mazzolai et al. [26] developed an octopus arm by arranging the transverse muscles and longitudinal muscles in parallel. The simultaneous contraction of the muscle tightened and antagonized the structure to achieve variable stiffness; however, independent control of the position and stiffness could not be achieved.

The flexible motion function, such as unidirectional rotation or bidirectional rotation, was generally used in previously developed pneumatic rotary actuators and joints. Still, they cannot maintain the position due to the lack of stiffness or damping adjustment device. Aiming to solve problems ensuing from the insufficient driving technology and enable maintaining the position of the pneumatic rotary joint, a new type of pneumatic bidirectional rotary flexible joint with controllable damping was developed. The joint was based on the antagonistic mechanism and works in coordination with two semicircular rotary actuators. The joint includes positive and antisymmetric distributions and a pneumatic brake, which can continuously adjust any position within $\pm 68.5^{\circ}$ and can retain the position in real time, depending on the task needs. Finally, the proposed joint can be used as the wrist of a mechanical arm to change and adjust the end claw posture. As such, it has broad application prospects and can be used in various industrial, medical, and service fields, which need flexible movement and braking maintenance equipment.

This paper is organized as follows: in Section 2, the structural design, fabrication process, and working principles describing the antagonistic pneumatic bidirectional rotary joint are provided. Furthermore, the static model of the actuator rotation angle and the brake torque model are established in Section 3. Models are then verified experimentally, as shown in Section 4. Furthermore, in the same section, the actuator deformation capacity, the brake performance, and the rotation angle and speed of the joint under no-load conditions are measured experimentally. A significant amount of experimental data is obtained, providing a basis for the subsequent joint control. Finally, the conclusions are given in Section 5.

\section{The Pneumatic Bidirectional Rotary Joint Structure and Function}

2.1. Joint Structure. As shown in Figure 1(a), the pneumatic bidirectional rotary joint is composed of clockwise and counterclockwise rotary actuators, pneumatic ring brake, end covers (upper and lower), sleeve, central shaft, and shell. The central axis position of the upper and lower end covers is defined using a stepped shaft, and shaft ends are mounted onto deep groove ball bearings. The outer bearing wall is connected to the upper and lower end covers. The clockwise and counterclockwise rotary actuators have identical structures, barring the installation phase difference of $180^{\circ}$. They are placed between the upper and the lower end cover. Furthermore, as shown in Figure 1(b), the air inlet end cover of the clockwise rotary actuator is installed on the vertical lower end cover plate using bolts, while the other end is installed on the rectangular sleeve baffle. The sleeve is connected with the central shaft using a key. The counterclockwise rotary actuator adopts the same installation method. A pneumatic ring brake is installed between the clockwise and the counterclockwise rotary actuators. Four threaded holes are evenly distributed along the brake housing circumference, and the ring brake is fixed on the shell by bolts. In addition to the rotary actuator and pneumatic ring brake, the remaining joint parts are also 


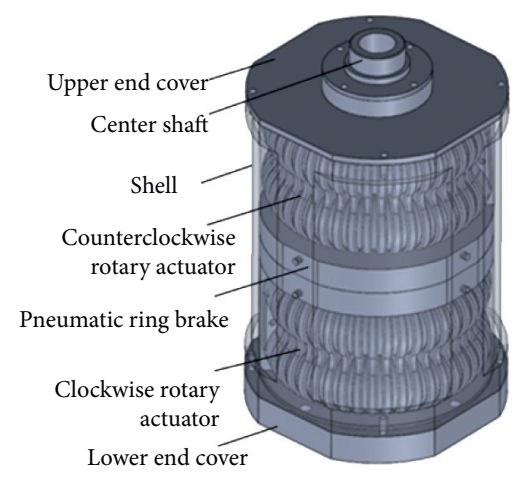

(a)

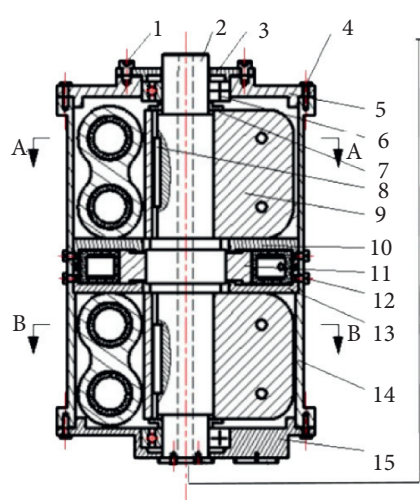

(b)

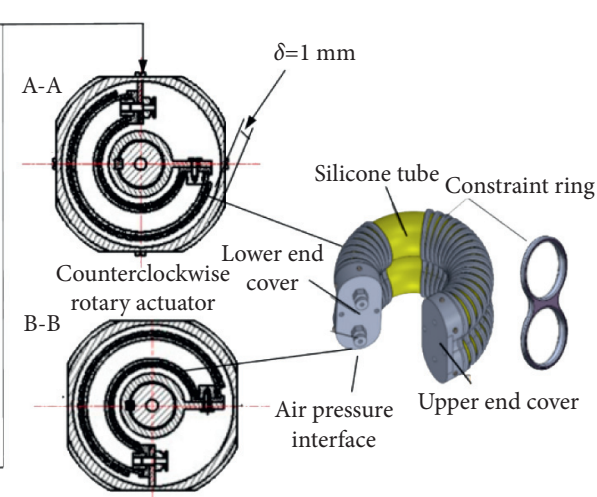

(c)

FIgURE 1: The structure of the antagonistic bidirectional rotary joint. (a) 3D model. (b) Structure diagram. (c) Rotary actuator 3D model.

made of aluminum alloy, and the total joint weight is approximately $1.5 \mathrm{~kg}$.

The rotary actuator consists of two curved artificial muscles connected in parallel, as shown in Figure 1(c). It should be noted that the closed cavity forms among the silicone tube, the constraint ring, the plug, and the air pressure interface. The outer side includes a set of eight wedge-shaped double-hole constraint rings. The elastic airbag is made of silicone tube, while the end cover and constraint rings are made of ABS using the rapid prototyping technology. The principal function of constraint rings is to limit the radial expansion of the silicone tube and ensure that the muscles are equidistant from the center.

The actuator manufacturing process is complex. Firstly, the two airbag ends are encapsulated using the upper and the air inlet plugs via the steel wire tightening method, aiming to form a closed cavity. To ensure airtightness, the constraint ring is preassembled for the ventilation experiment. The air inlet plug center is a threaded through-hole, which can be connected with the air pressure interface using screws. Simultaneously, the air inlet plug side surface includes a threaded hole, which is used to connect it with the lower end cover by using screws. Secondly, the eight wedge-shaped double-hole constraint rings are set in sequence. Finally, the assembly is completed by a screw connection between the threaded hole at the upper plug end and its cover.

When compressed air is introduced into the rotary actuator, the inner wall of the silicone tube expands under pressure. The constraint rings are set on the outer silicone tube side to limit the radial expansion, primarily to generate axial force and push the sleeve to achieve a circular motion. When the air pressure is removed, the rotary actuator returns to its original state due to the elastic energy contained in the silicone tube.

As shown in Figure 2(a), the pneumatic ring brake is assembled of the upper and lower brake housings, the annular airbag, the friction plate, and the limit plate. The upper and lower housings are cylindrical shell structures with annular bosses and four equally spaced limit grooves at the center. A limiting surface is placed on both upper and lower fan-shaped friction plates and is matched with the annular brake housing boss to restrict its radial movement. At the same time, the limit plate also limits the circumferential rotation of the friction plate. The friction plate is installed on the inner airbag wall via an interference fit, while the annular airbag is installed within the brake housing. The annular airbag is made of Dragon Skin 30 liquid silicone material by split casting, as shown in Figure 2(b). When compressed air is introduced into the airbag, its inner wall expands under pressure. Since the vertical direction and the outer brake side are restricted by the joint shell, the airbag inflates inwards, pushing the fan friction plate in the axial direction. With the increase in pressure, the fan-shaped friction plate comes into contact with the shaft and produces friction, achieving the braking function. The braking force generated by the brake is proportional to the air pressure within the annular airbag.

2.2. Motion Principle of the Joint. Figure 3 shows the motion principle diagram of the antagonistic pneumatic bidirectional rotary joint. The clockwise and counterclockwise actuators represent a pair of antagonistic muscles. During the installation, the clockwise and counterclockwise actuators require input pressure $p_{0}$ as a preload to rotate the joint to an angle $\theta_{0}$, as shown in Figure 3(b). By adjusting the air pressure values of the two sets of actuators $\left(p_{1}=p_{2}, p_{3}=p_{4}, p_{1}, p_{2}, p_{3}\right.$, and $p_{4}$ are the artificial muscle pressures), the joint can be moved in a circular motion in both directions. The specific implementation forms are as follows: increasing the clockwise actuator air pressure $\left(p_{1}=p_{2}=p_{0}+\Delta p\right)$, decreasing the counterclockwise actuator air pressure $\left(p_{3}=p_{4}=p-\Delta p\right)$, and increasing the clockwise actuator air pressure while reducing the counterclockwise once $\left(p_{1}=p_{2}=p_{0}+\Delta p\right.$ and $p_{3}=p_{4}=$ $\left.p_{0}-\Delta p\right)$. All of the three above-listed forms result in the clockwise circular joint motion, as shown in Figure 3(c). The counterclockwise circular joint motion is achieved identically $\quad\left(p_{1}=p_{2}=p_{0}-\Delta p, \quad p_{3}=p_{4}=p+\Delta p \quad\right.$ and $\left.p_{1}=p_{2}=p_{0}-\Delta p, p_{3}=p_{4}=p_{0}+\Delta p\right)$, as shown in Figure $3(\mathrm{a})$. When the joint reaches the designated position, the brake is supplied with the air pressure to brake and maintain the position. 


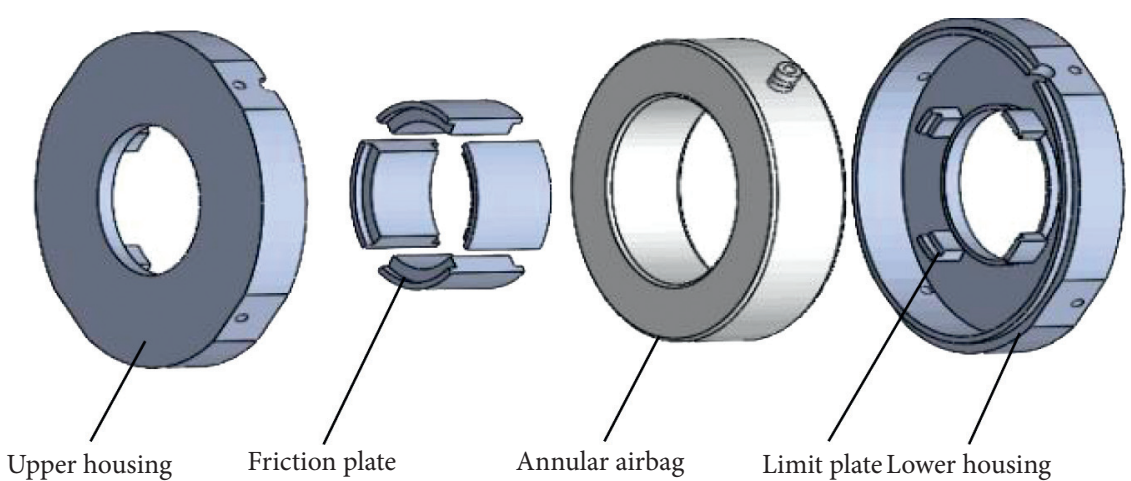

(a)

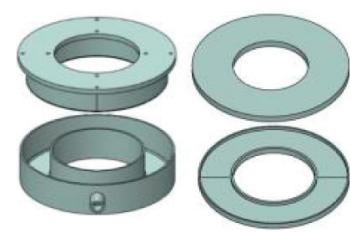

(1) 3D Printed airbag mold

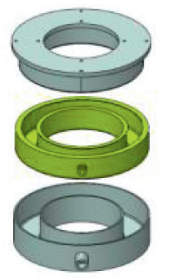

(2) Pouring the airbag cavity

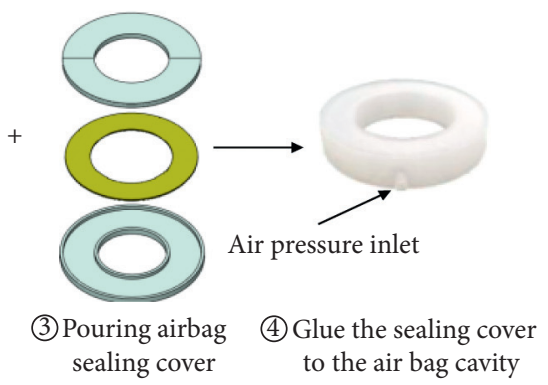

(b)

FIGURE 2: Brake structure and the manufacturing airbag process. (a) Exploded view (brake). (b) The airbag manufacturing process.

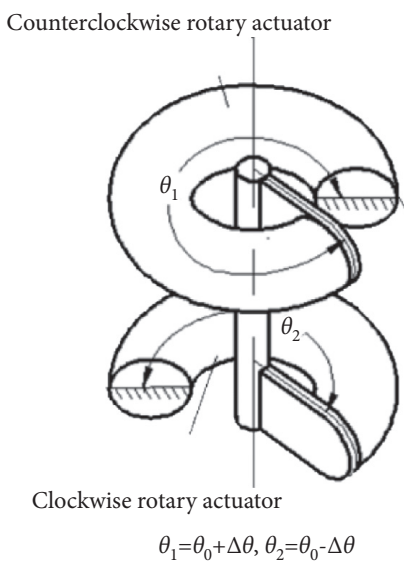

(a)

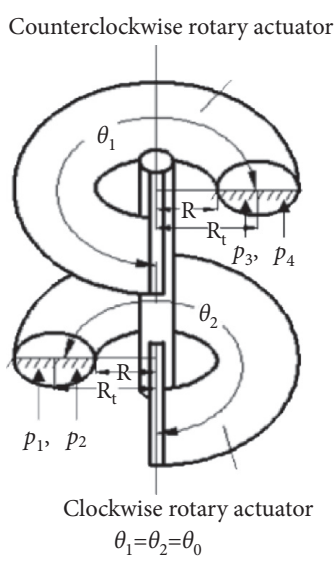

(b)

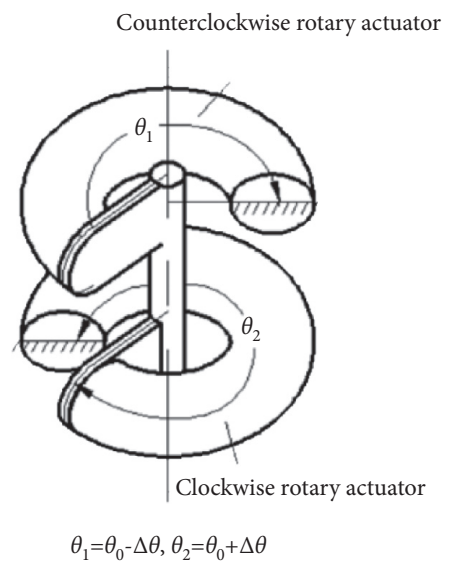

(c)

Figure 3: Schematic diagram of the joint movement. (a) Counterclockwise rotation. (b) Antagonistic initial state. (c) Clockwise rotation.

\section{Static Model}

3.1. Wall Thickness Analysis of the Artificial Rotary Actuator Muscle. The initial actuator angle is defined as $\theta$, while the increment angle is $\Delta \theta$ under the air pressure action. The deformation analysis of a single actuator is shown in Figure 4. Length $\mathrm{O}_{1} \mathrm{O}_{2}$ represents the central joint axis; $\mathrm{O}_{1} \mathrm{O}_{4}$ and $\mathrm{O}_{1} \mathrm{O}_{3}^{\prime}$ are the joint radii of rotation. Assuming that the length is constant during the deformation process, their values were set to $R_{t}$. Planes $\mathrm{O}_{1} \mathrm{O}_{2} E \mathrm{EO}_{4}$ and $\mathrm{O}_{1} \mathrm{O}_{2} \mathrm{DIO}_{3}^{\prime}$ are the actuator end covers, and the angle between the end covers is the sum of the initial angle $\theta$ and the increment angle $\Delta \theta$ (designated as $\left.\angle O_{4} O_{1} O_{3}^{\prime}\right)$. The plane $O_{4} O_{1} O_{3}^{\prime}$ is a cross section through the actuator axis; since it is parallel to $E O_{2} D^{\prime}$, angle $\angle E O_{2} D^{\prime}=\angle O_{4} O_{1} O_{3}^{\prime}$ can be obtained. Furthermore, $E D^{\prime}$ is the curve where the plane $E O_{2} D^{\prime}$ intersects with the actuator. $O_{3}^{1} D^{\prime}=r_{t}$ is defined as the average artificial muscle radius, while the angle $\angle B O_{3}^{\prime} D^{\prime}$, located on the free actuator end face, is defined as $\Phi$ (its value ranges between 0 and $2 \pi$ ). Finally, $O_{3}^{1} D^{\prime}=r_{t}$ is defined as the average radius of the artificial muscle, while $E D^{\prime}$ curve length is defined as $\lambda_{\phi}$. 


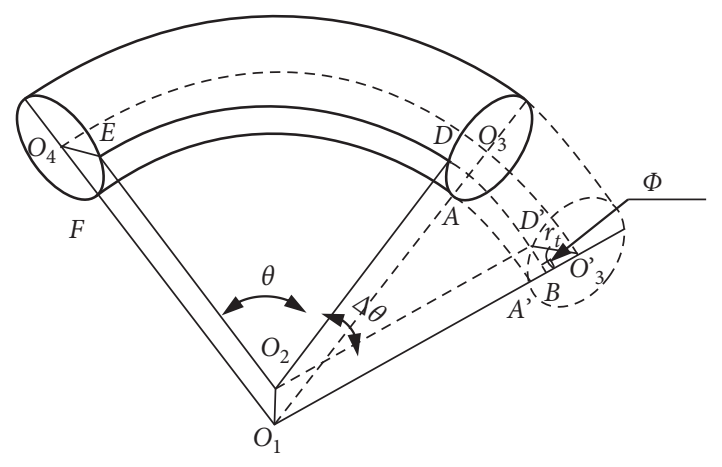

Figure 4: Actuator deformation analysis.

As shown in Figure 4, $B D^{\prime} \perp O_{1} O_{3}^{\prime}$. Hence, $B O_{3}^{\prime}$ in the right triangle $\triangle B O_{3}^{\prime} D$ ' can be expressed as follows:

$$
B \mathrm{O}_{3}^{\prime}=r_{t} \cos \Phi .
$$

According to the geometric relationship, $O_{1} O_{2} / / B D^{\prime}$, meaning that $\mathrm{O}_{2} \mathrm{D}$ ' can be expressed as

$$
\mathrm{O}_{2} D \prime=O_{1} O_{3}^{\prime}=R_{t}-r_{t} \cos \Phi .
$$

In sector $\mathrm{O}_{2} D \prime E$, the arc length $\lambda_{\Phi}$ is obtained as follows:

$$
\lambda_{\Phi}=O D^{\prime}(\theta+\Delta \theta)=\left(R_{t}-r_{t} \cos \Phi\right)(\theta+\Delta \theta) .
$$

Therefore, the silicone tube wall thickness $T_{\Phi}$ at angle $\Phi$ can be calculated using

$$
T_{\Phi}=\frac{\lambda_{t} T_{t}}{\lambda_{\Phi}}=\frac{\lambda_{t} T_{t}}{\left(R_{t}-r \cos \Phi\right)(\theta+\Delta \theta)},
$$

where $\lambda_{t}$ is the length of the initial effective deformation arc of the rotary actuator silicone tube and $T_{t}$ is the initial wall thickness of the rotary actuator silicone tube.

3.2. Static Model of the Actuator Rotation Angle. As shown in Figure 5, the torque balance analysis of the free actuator end (the end fixed with a sleeve) can be written as

$$
\sum_{i=1}^{2} M_{p}=M+\sum_{i=1}^{2} M_{n}+\sum_{i=1}^{2} M_{f}
$$

where $M_{p}$ is the driving torque generated by the actuator under the air pressure, $M$ is the external torque received during the actuator rotation, $M_{n}$ is the silicone tube impedance torque, and $M_{f}$ is the friction torque generated by the central shaft and the bearing during the rotation.

The driving torque can be obtained from the torque formula:

$$
\mathrm{d} M_{p}=L_{p} \mathrm{~d} F_{p}=L_{p} p \mathrm{~d} A_{1}=L_{p} p r \mathrm{~d} r \mathrm{~d} \Phi,
$$

where $L_{p}$ is the torque arm of force $\mathrm{d} F_{p}$ relative to the central axis.

The torque arm $L_{p}$ calculation method is similar to equation (3) and can be obtained as follows:

$$
L_{p}=R_{t}-r_{t} \cos \Phi \text {. }
$$

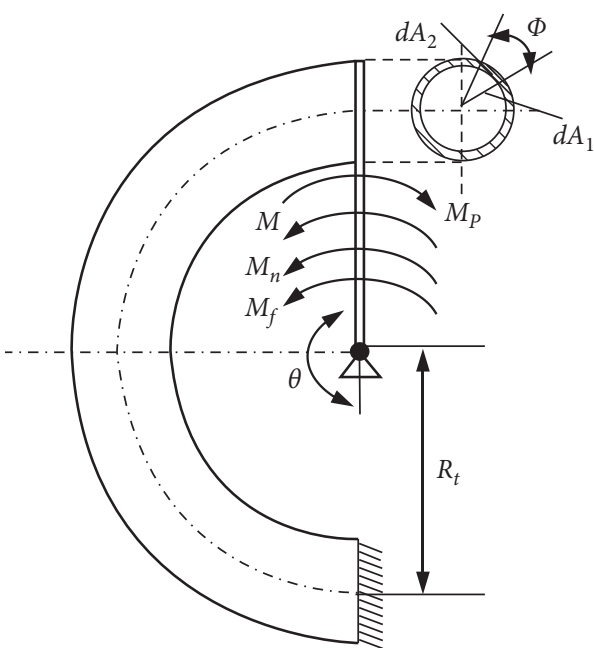

FigURe 5: Actuator torque analysis.

By combining equations (6) and (7), the actuator driving torque can be found as follows:

$$
M_{p}=\int \mathrm{d} M_{p}=2 \int_{0}^{\pi} \int_{0}^{r_{t}} p\left(R_{t}-r \cos \Phi\right) r \mathrm{~d} r \mathrm{~d} \Phi=r_{t}^{2} R_{t} \pi p,
$$

$\mathrm{d} F_{a}=\sigma \mathrm{d} A_{2}=E \frac{\lambda_{\Phi}-\lambda_{t}}{L_{t}} T_{\Phi} r_{t} \mathrm{~d} \Phi$

where $\sigma$ is the silicone tube stress and $E$ is the elastic modulus of the silicone tube.

Combining expressions (3), (4), and (9) yields the actuator silicone tube impedance torque:

$$
\begin{aligned}
M_{n}= & \int L_{a} \mathrm{~d} F_{a}=2 E T_{t} r_{t} \int_{0}^{\pi}\left(R_{t}-r_{t} \cos \Phi\right) \\
& \frac{(\theta+\Delta \theta)\left(R_{t}-r_{t} \cos \Phi\right)-\lambda_{t}}{(\theta+\Delta \theta)\left(R_{t}-r_{t} \cos \Phi\right)} \mathrm{d} \Phi, \\
= & 2 \pi E T_{t} r_{t}\left(R_{t}-\frac{\lambda_{t}}{\theta+\Delta \theta}\right) .
\end{aligned}
$$

The friction torque between the central shaft and the bearing is

$$
M_{f}=\frac{\mu P d}{2},
$$

where $\mu$ is the bearing friction coefficient, $P$ is the bearing load, and $d$ is the nominal inner bearing diameter.

By substituting equations (8), (10), and (11) into (5), the actuator increment angle can be calculated as follows:

$$
\Delta \theta=\frac{4 \pi E T_{t} r_{t} \lambda_{t}}{4 \pi E T_{t} R_{t} r_{t}+M+\mu P d-2 \pi p R_{t} r_{t}^{2}}-\theta,
$$

where $\theta=\lambda_{t} / R_{t}$ is the initial actuator rotation angle.

Equation (12) describes the static angle model when a single actuator is elongated. According to the working principle of the antagonistic pneumatic bidirectional 
rotary joint, one actuator will always be in a compressed state. Since the silicone tube compression deformation cannot be controlled, the impedance torque of the compressed actuator at various air pressures was measured experimentally.

3.3. Brake Braking Torque Model. When compressed air is introduced into the brake, the airbag expands under the air pressure action, pushing the friction plate to the central shaft generating friction; thus, the braking is achieved, as shown in Figure 6.

Before the brake torque modeling, the following basic assumptions were made:

(1) The friction plate is made of aluminum alloy and is rigid; that is, the influence of the structural elastic deformation on the contact area is not considered.

(2) The positive pressure acting on the friction plate surface is evenly distributed along the width direction.

(3) The contact between the inner surface of the friction plate and the outer surface of the central shaft is a cylindrical surface. The radius of curvature of the two working surfaces is equal.

An arbitrary value was selected for the transverse unit area of a small block for the external friction plate working surface, and its size was expressed as

$$
\mathrm{d}_{s}=b R_{1} \mathrm{~d}_{\alpha}
$$

where $d_{s}$ is the transverse unit area, $R_{1}$ is the inner radius of the annular airbag surface, and $b=R_{1}-R_{2}$ is the friction plate width. Furthermore, $R_{1}$ is the outer radius of the friction plate surface, $R_{2}$ is the inner radius of the friction plate surface, and $d_{\alpha}$ is the transverse unit wrap angle.

After the airbag is filled using the compressed air, the normal force acting on the friction plate microelement area can be considered as

$$
\mathrm{d} F_{N}=p b R_{1} \mathrm{~d}_{\alpha}
$$

where $\mathrm{d} F_{N}$ is the normal force acting on the microelement area.

The braking torque generated by the friction can be calculated as follows:

$$
\begin{aligned}
T_{\mu 1} & =\int_{-\beta / 2}^{\beta / 2} \mathrm{~d} F_{f} R_{3}=\int_{-\beta / 2}^{\beta / 2} \mu \mathrm{d} F_{N} R_{3}=\int_{-\beta / 2}^{\beta / 2} \mu p b R_{1} R_{3} \mathrm{~d}_{\alpha} \\
& =\mu p b R_{1} R_{3} \beta,
\end{aligned}
$$

where $T_{\mu 1}$ is the braking torque generated by a single friction plate, $\mathrm{d} F_{f}=\mu \mathrm{d} F_{N}$ represents the friction acting on the microelement area, $R_{3}$ is the radius of the contact between the friction plate and the central shaft, $\mu$ is the friction coefficient between the friction plate and the central shaft, and $\beta$ is the wrap angle corresponding to a single friction plate.
The structures of four brake friction plates are identical; therefore, the total braking torque can be obtained as follows:

$$
T_{\mu}=\sum_{i=1}^{4} T_{\mu i}=4 \mu p b R_{1} R_{3} \beta,
$$

where $T_{\mu}$ is the braking torque generated by the brake.

\section{Experiment and Joint Analysis}

The mechanical properties of the actuator and brake, as critical components of the pneumatic bidirectional rotary joint, directly affect joint characteristics. The static joint experimental system is shown in Figure 7. In addition to the joint body and air pressure control system, this experimental system also includes a data acquisition system. A rotation angle sensor (model: HWT101DT) was installed on the top of the joint to measure its angle and angular velocity. The sixaxis force sensor (model: WEF-6A200-4-RC5-A) was used to obtain the change of driving torque with the air pressure after the rotary actuator contacts the target at various rotation positions. The relationship between the braking force and air pressure was measured using a load sensor (model: $\mathrm{XJH}-10$ ). The air pressure sensor (model: ISE20-P-M-M5) could measure the actuator air pressure. The specifications of the sensor used in the experiment are shown in Table 1. The joint prototype with an overall length of $180 \mathrm{~mm}$, a diameter of $110 \mathrm{~mm}$, and a mass of $1500 \mathrm{~g}$ was manufactured. The specific structural parameters of both the actuator and the brake are given in Tables 2 and 3.

4.1. Mechanical Performance Experiment. When the compressed air was simultaneously introduced into the actuator airbags, the actuator rotation angle can be measured by the rotation angle sensor for various air pressures (model: HWT101DT). Figure 8 shows changes in bending angles under the inflation and deflation process.

As shown in Figure 8, the actuator angle increases nonlinearly with the increase in air pressure. Due to the hysteresis phenomenon of the pneumatic artificial muscle, the actuator angle in the deflation process is slightly higher than during the inflation process. Before reaching the pressure of $0.04 \mathrm{MPa}$, the rotation angle is small, mainly due to the airbag inflation and expansion. It is necessary to overcome its resistance and to eliminate the installation gap between constraint rings and the airbag. When the pressure reaches $0.16 \mathrm{MPa}$, the rotation angle speed increases slowly. Finally, at $0.24 \mathrm{MPa}$, the maximum actuator angle was $142.9^{\circ}$.

The theoretical rotation angle can be obtained by substituting the actuator parameters into equation (12). Figure 9 shows the comparison between the theoretical curves and experimental data.

Based on the comparison between the two, it can be seen that, before reaching $0.18 \mathrm{MPa}$, the trends of theoretical rotation angle calculation results are the same as the experimental data. Therefore, the established theoretical model reflects the static angle characteristics of the actuator. During 


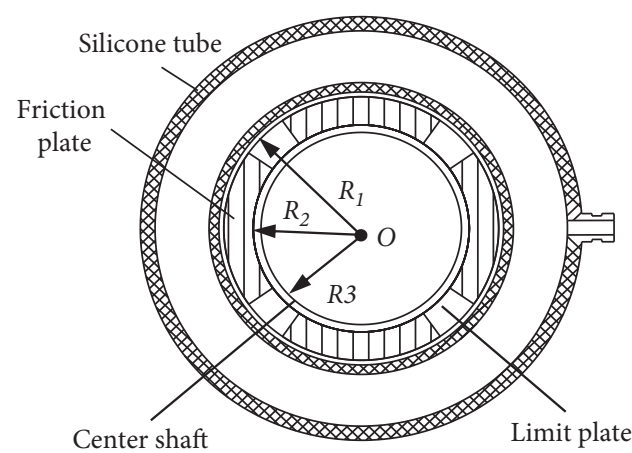

(a)

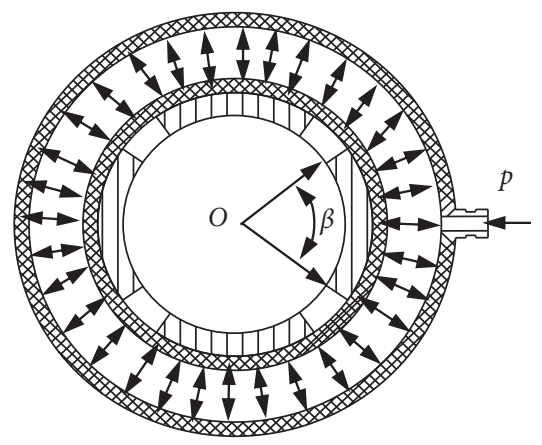

(b)

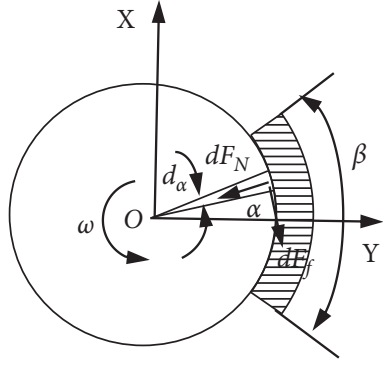

(c)

FIgURE 6: The brake working mechanism.

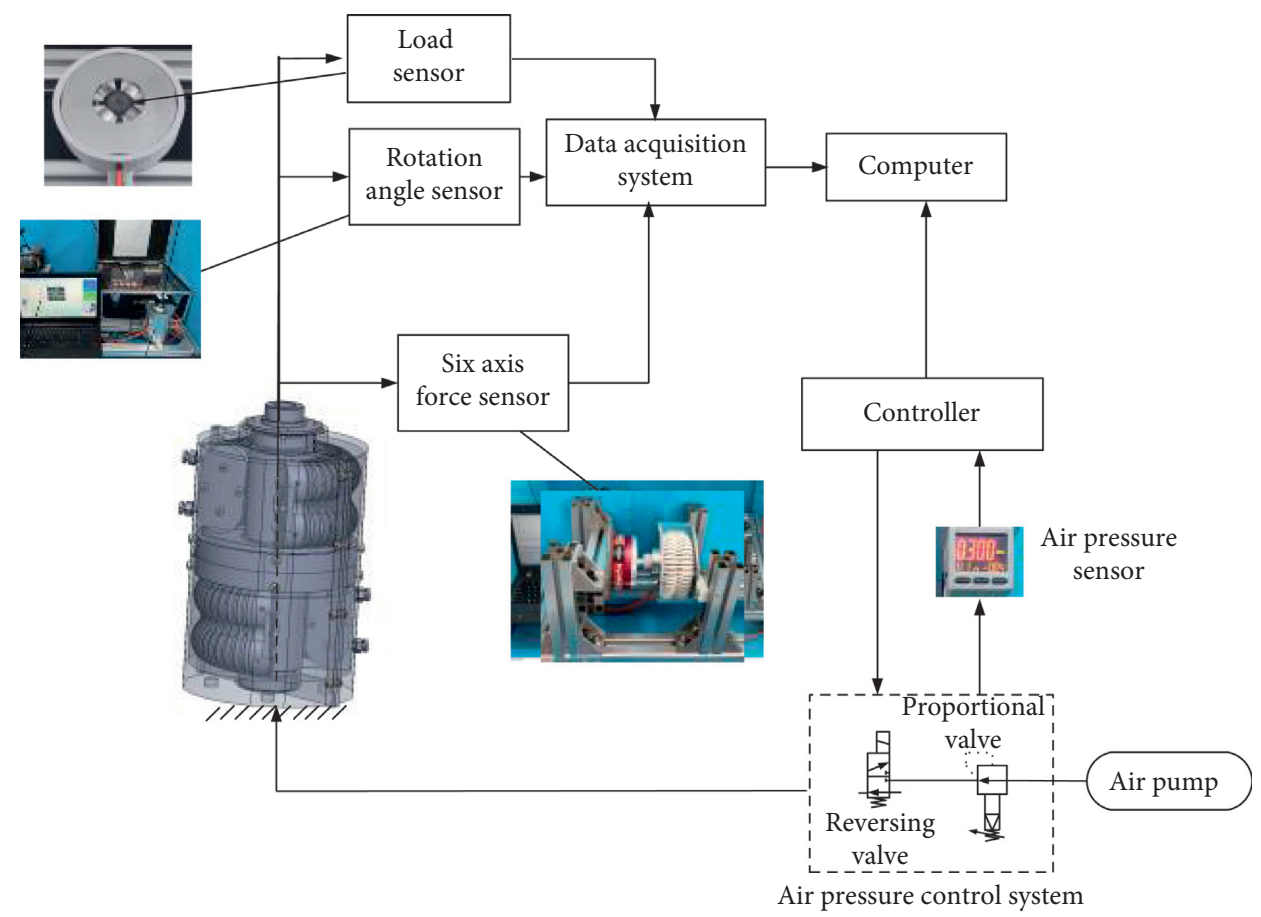

FIgURE 7: Static experimental system of the joint.

TABLe 1: Sensor specifications.

\begin{tabular}{lccr}
\hline Name & Model & Rated range & Accuracy \\
\hline Air pressure sensor & ISE20-P-M-M5 & $-0.1-1.0 \mathrm{MPa}$ & $0.001 \mathrm{MPa}$ \\
Six-axis force sensor & WEF-6A200-4-RC5-A & Fx, Fy, Fz: $200 \mathrm{~N}$ & Transverse sensitivity below 5\% F.S \\
& & Mx, My, Mz: $4 \mathrm{Nm}$ & $0.05^{\circ}$ \\
Rotation angle sensor & HWT101DT & Angle: $\pm 180^{\circ}$ & $\pm 0.5 \%$ F.S \\
Load sensor & XJH-10 & Angular velocity: $\pm 2000^{\circ} / \mathrm{s}$ & $10-200 \mathrm{~kg}$ \\
\hline
\end{tabular}

the initial stage of the pressure and actuator rotation angle experimental curves, the rotation angle increases slowly. When the air pressure reaches a certain value, the rotation angle increases rapidly. The main reasons for this phenomenon are as follows: on the one hand, the silicon tube is to the hyperelastic body, which has a certain viscosity. On the other hand, the actuator does not deform and bends completely according to its original arc after inflation. At the same time, due to the influence of manufacturing defects, deviations in the actuator rotation trajectory and radial 
TABLE 2: Structural parameters of the actuator.

\begin{tabular}{lcc}
\hline Parameter & Numerical value & Unit \\
\hline The silicone tube radius & 10 & $\mathrm{~mm}$ \\
The silicone tube initial wall thickness & 2 & $\mathrm{~mm}$ \\
The silicone tube elastic modulus & 1.116 & $\mathrm{MPa}$ \\
The distance between the silicone tube and the central shaft axes & 100 & $\mathrm{~mm}$ \\
The initial effective actuator deformation arc length & 110 & $\mathrm{~mm}$ \\
The total effective actuator deformation arc length & 56 & $\mathrm{~mm}$ \\
The actuator height & 180 & $\mathrm{~mm}$ \\
The initial angle of the actuator &
\end{tabular}

TABLE 3: Structural parameters of the brake.

\begin{tabular}{lcc}
\hline Parameter & Numerical value & unit \\
\hline The outer surface radius of the annular airbag & 45 & $\mathrm{~mm}$ \\
The inner surface radius of the annular airbag & $38 \mathrm{~m}$ \\
The annular airbag wall thickness & 22.5 & $\mathrm{~mm}$ \\
The inner friction plate surface radius & 28 & $\mathrm{~mm}$ \\
The outer friction plate surface radius & 14 & $\mathrm{~mm}$ \\
The wrap angle of the friction plate & 22 & $\mathrm{~mm}$ \\
The width of the friction plate & $\mathrm{mm}$ \\
The radius of the contact between the friction plate and the central shaft & \\
\hline
\end{tabular}

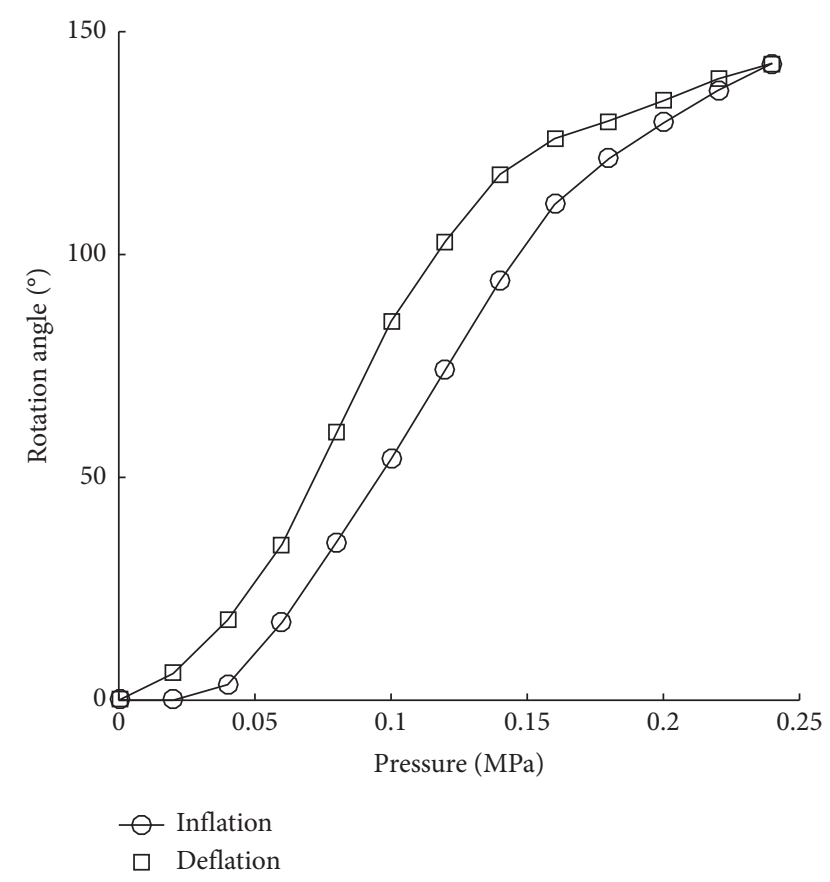

FIGURE 8: Actuator inflation/deflation process.

expansion of the silicone tube were ignored while establishing the theoretical model. Hence, the obtained theoretical model is relatively simple and has a certain error compared to the experimental data.

Above $0.18 \mathrm{MPa}$, the experimental data values were lower than the theoretical ones. The main reason for such a behavior was the friction force generated by the contact between the constraint rings and the shell, which caused the

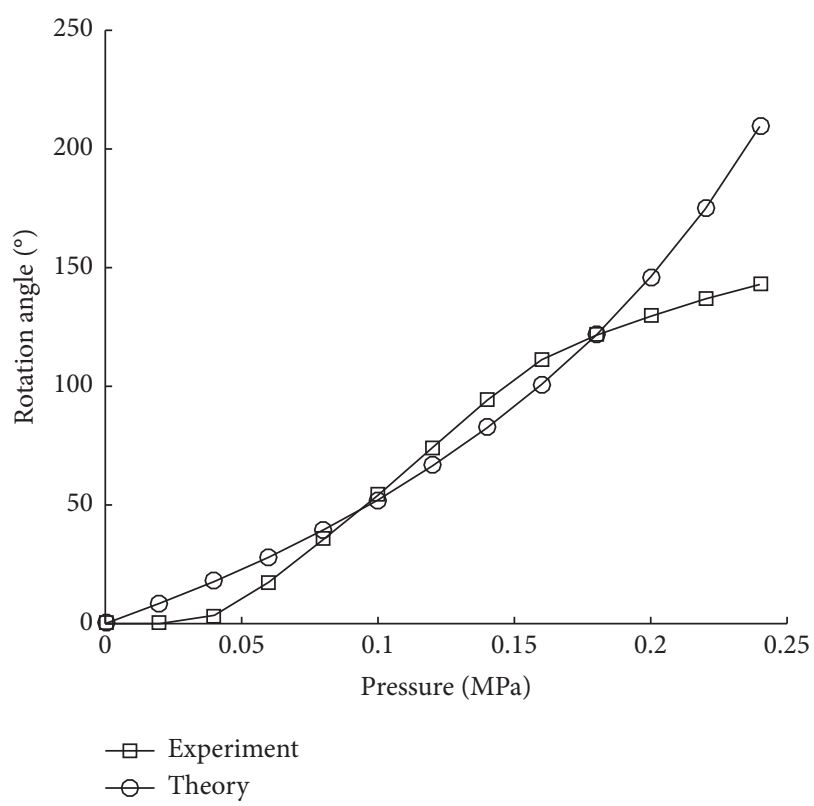

Figure 9: Comparison between the experimental and theoretical rotation angles.

actuator rotation angle to increase slowly, making it lower than the theoretical data.

The six-axis force sensor (model: WEF-6A200-4-RC5-A) was used to obtain the driving torque curve change caused by the air pressure after the rotary actuator comes into contact with the target at different rotation positions (see Figure 10). During the experiment, the actuator freely rotates to different limit planes and is locked with a coupling. 


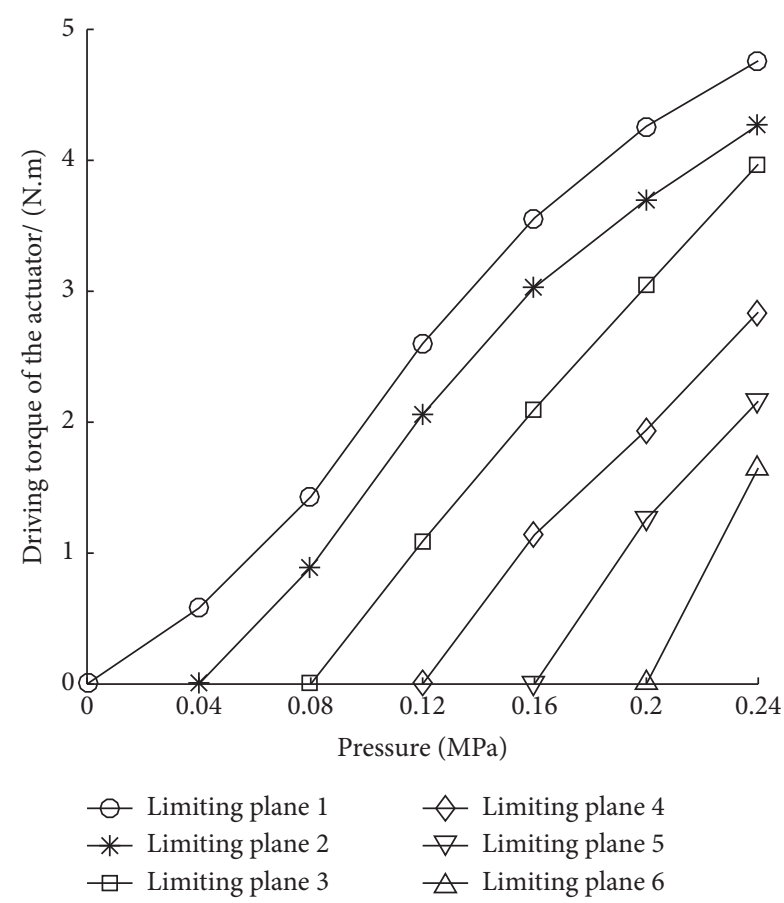

Figure 10: Comparison curve of driving torque in different limiting planes.

The air pressure is then increased to measure the torque; the increment of $0.04 \mathrm{MPa}$ was used.

As shown in Figure 10, the joint driving torque linearly increases with the air pressure. Therefore, the driving torque can be controlled by adjusting the actuator muscle air pressure. The difference is that the actuator driving capacity to different limit planes varies. The closer the limit plane is to the actuator rotation starting point, the stronger its driving capacity will be. Therefore, the actuator has the strongest driving capacity at the plane 1 limit. The maximum driving torque reaches $4.76 \mathrm{Nm}$ when the air pressure is $0.24 \mathrm{MPa}$.

Using the same experimental device, the impedance torque generated by the actuator was measured at different rotation angles. During the experiment, the actuator was first freely deformed to the maximum rotation angle (for various air pressures) and locked with a coupling. Next, the actuator was completely depressurized, and its impedance torque was measured at a preselected rotation angle, as shown in Figure 11. As shown in Figure 11, the impedance torque increases with the rotation angle. The maximum rotation angle was $142.9^{\circ}$, with the corresponding actuator impedance torque of $0.92 \mathrm{Nm}$.

4.2. Mechanical Performance Experiment of the Brake. When the compressed air is introduced into the brake, the airbag expansion pushes the friction plate to the central shaft, generating positive pressure. The positive pressure at the contact point can be measured using the load sensor (model: XJH-10). Figure 12 shows the change of the positive pressure generated by the brake depending on the air pressure. It can be seen that the positive brake pressure

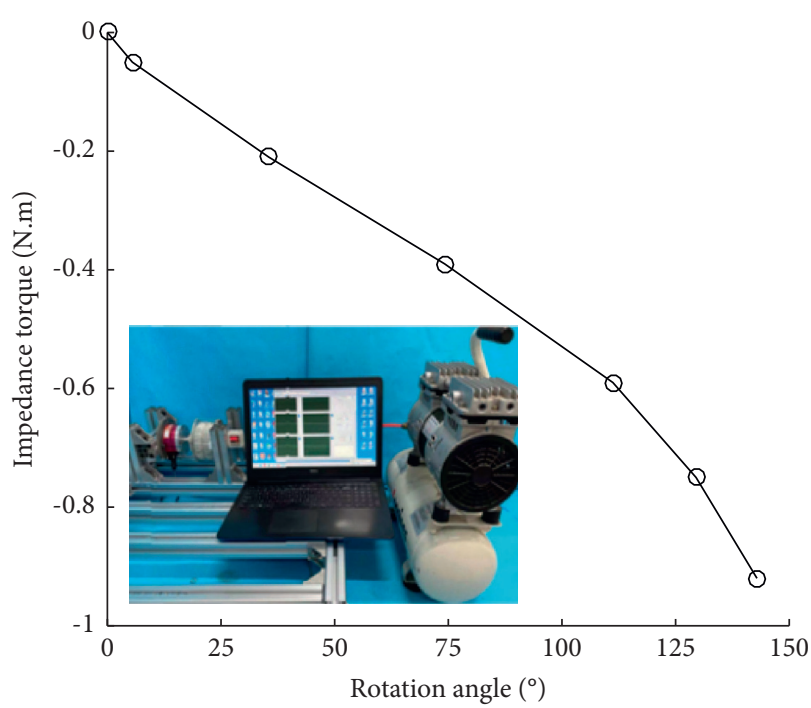

FIgURE 11: Relationship between impedance torque and rotation angle.

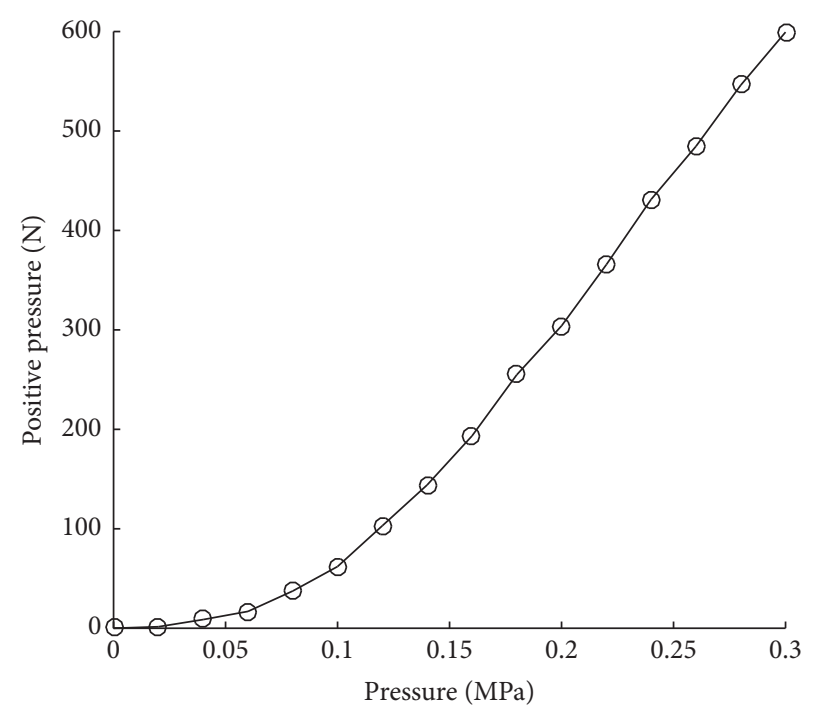

FIGURE 12: Relationship between the positive brake pressure and the air pressure.

increases nonlinearly with the air pressure. The maximum positive brake pressure was $598.9 \mathrm{~N}$ and was obtained for the $0.30 \mathrm{MPa}$ air pressure.

Figure 13 provides the schematic diagram of the experimental braking torque. The lower end joint cover is fixed, and a groove is made on the output end side of the central shaft to hang the beaker. Simultaneously, a rotation angle sensor was installed on the end surface of the central shaft to monitor its angular acceleration. During the experiment, the brake air pressure was gradually increased through the precision pressure reducing valve (the air pressure increment was $0.04 \mathrm{MPa}$ ). The brake load capacity was tested by continuously injecting water into the beaker. When the angle sensor detected a sudden change in the angular acceleration of the central shaft, water injection was 


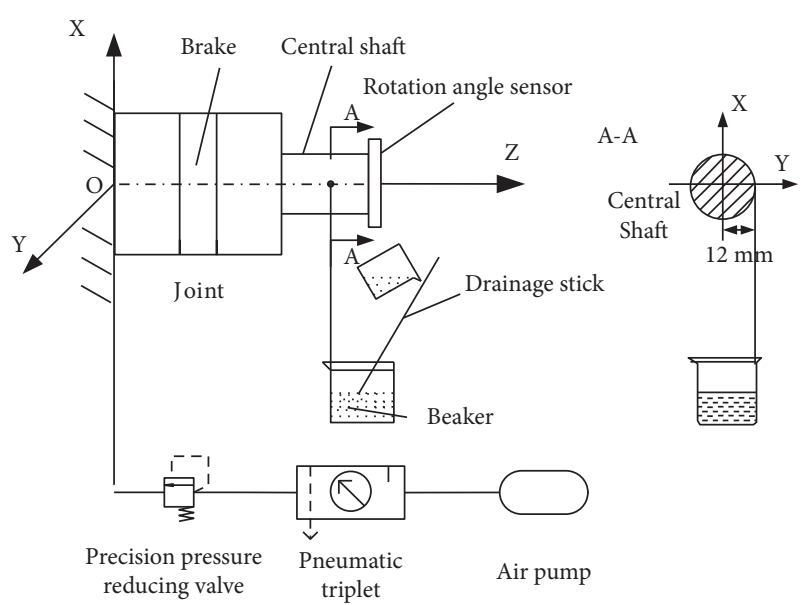

FIGURE 13: Experimental schematic diagram of the braking torque.

immediately stopped. The weight of water in the beaker was measured, recorded, and taken as the maximum load that the brake can withstand for given air pressure. The maximum braking torque provided by the brake for a given air pressure was obtained using the torque calculation formula. Additionally, theoretical braking torque data were obtained by substituting the structural brake parameters into equation (14). Finally, Figure 14 illustrates the comparison between theoretical and experimental data.

Furthermore, as can be seen in Figure 14, the theoretical data are consistent with their experimental counterparts, and the maximum error was $8.6 \%$. Thus, the theoretical model's correctness was verified to a certain extent. The braking torque increased approximately linearly with the air pressure. The maximum braking torque was $4.21 \mathrm{Nm}$ and was obtained for the air pressure of $0.32 \mathrm{MPa}$.

4.3. Mechanical Performance Experiment of the Joint. A single actuator cannot achieve the bidirectional rotation motion; for this reason, two rotary actuators were used to form an antagonistic joint, achieving the bidirectional rotation. By adjusting the air pressure of the clockwise and counterclockwise actuator, the joint can be controlled and rotated within $\pm 60.5^{\circ}$ (see Figure 15). As is evident from Figure 15 , the rotation angle $0^{\circ}$ was taken as the dividing line and is proportional to the pressure difference between the actuators. When $p_{1}=p_{2}=p_{3}=p_{4}$, the joint rotation angle is $0^{\circ}$. Furthermore, when the pressure difference between the actuators reaches the maximum value $\left(p_{1}=p_{2}=0.24, p_{3}=p_{4}=0 \quad\right.$ or $\left.p_{1}=p_{2}=0, p_{3}=p_{4}=0.24\right)$, the rotation angle has a maximum value of $60.5^{\circ}$.

To determine the optimal joint installation preload, the symmetrical pressure experiment was carried out (clockwise actuator varies from 0 to $0.14 \mathrm{MPa}$, while the counterclockwise actuator varies from 0.14 to $0 \mathrm{MPa}$ ). The air pressure increment was set to $0.02 \mathrm{MPa}$, and the sum of absolute values of forward and reverse rotation angles was taken as the evaluation index used to obtain the relation

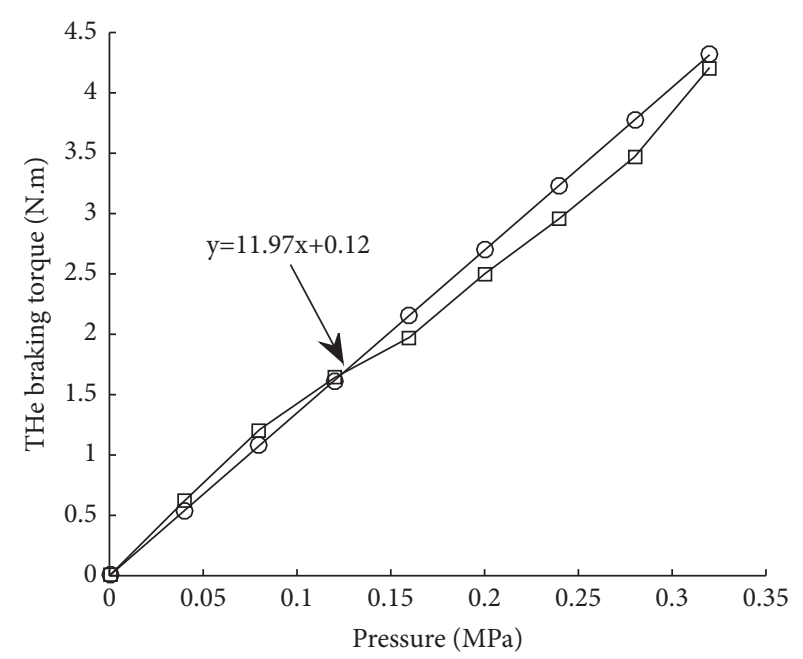

$$
\begin{aligned}
& \square \text { Exprement } \\
& \bigcirc-\text { Theory }
\end{aligned}
$$

FIgURE 14: The relationship between the braking torque and air pressure.

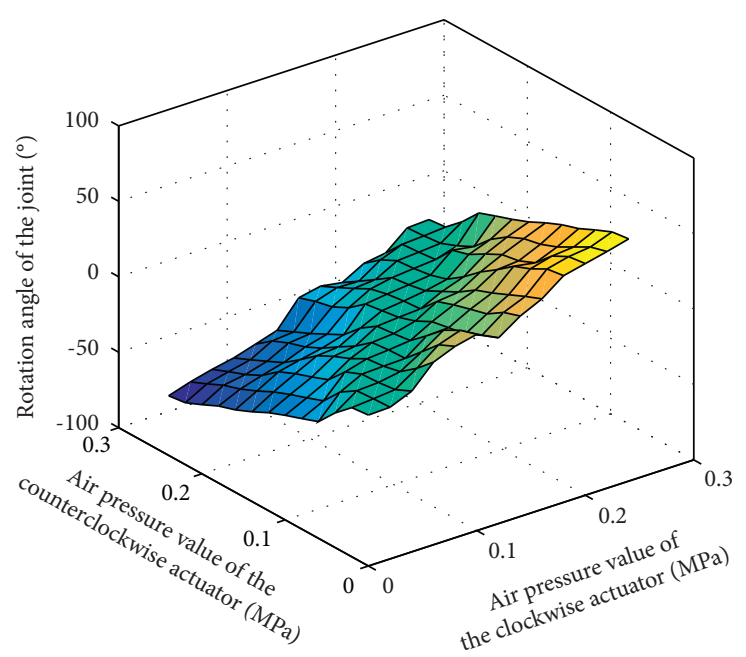

FIgURE 15: The relationship between the rotation angle and air pressure.

between the joint rotation angle and the air pressure, as shown in Figure 16.

As shown in Figure 16, the sum of the joint rotation angle absolute values firstly increases with the increase in air pressure, which is followed by a decrease. At $0.12 \mathrm{MPa}$, it reaches the peak value of $119.8^{\circ}$; therefore, the optimal joint installation preload is $0.12 \mathrm{MPa}$.

After adjusting the actuator air pressures, a six-dimensional force sensor was used to measure the joint driving torque at limit plane 1 . Figure 17 shows variations of joint driving torque surfaces with the air pressure. The joint driving torque is proportional to the pressure difference between the actuators, and the driving torque varies from 0 to $4.76 \mathrm{Nm}$. When the two actuators are supplied with the same air pressure, the driving torque is $0 \mathrm{Nm}$. The maximum 


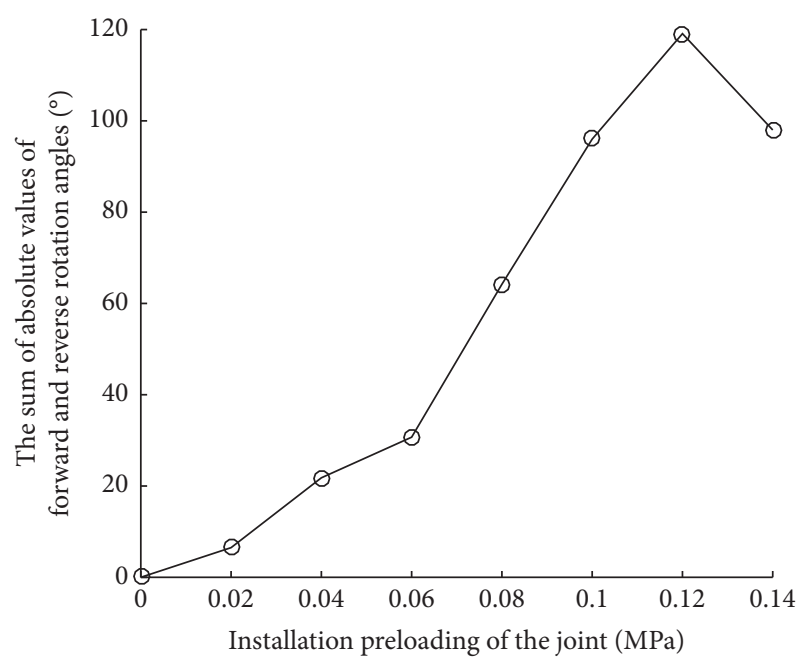

FIGURE 16: The relationship between the sum of rotation angles and the preload.

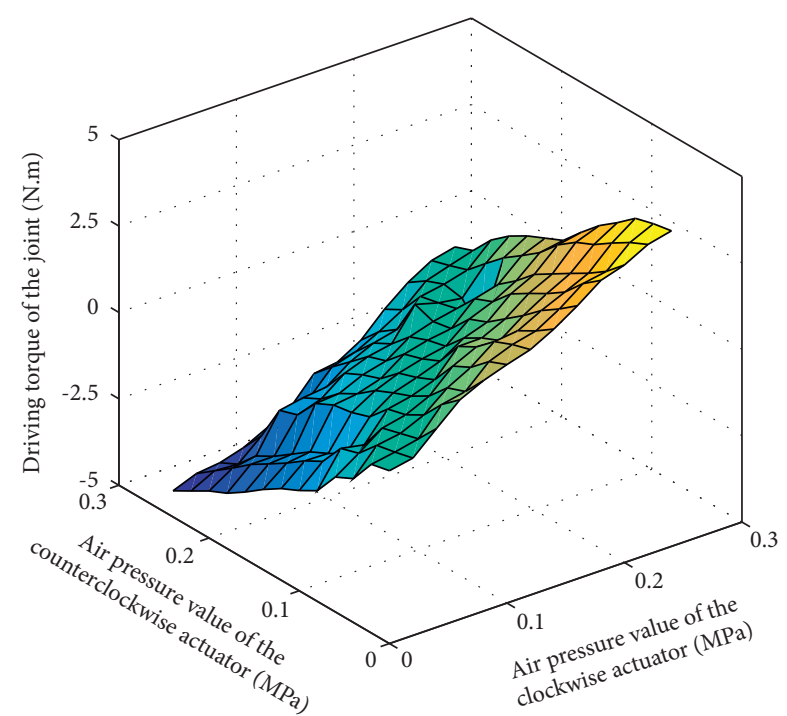

FIGURE 17: The relationship between the driving torque of the joint and air pressure.

driving torque is $4.76 \mathrm{Nm}$ when the pressure difference between the two actuators is $0.24 \mathrm{MPa}$.

4.4. Motion Performance Experiment of the Joint. To eliminate the effects of gravity, the joint was installed vertically with the optimal antagonistic preload of $0.12 \mathrm{MPa}$. Under the no-load condition, the joint response time during the forward and reverse motions was measured using a step signal. Firstly, during the experiment, the counterclockwise actuator was provided with air pressure excitation, while the clockwise actuator was relieved. When the joint was rotated counterclockwise to reach the stable angle, the counterclockwise actuator was relieved. Next, the clockwise actuator was charged, and the joint returned to the initial

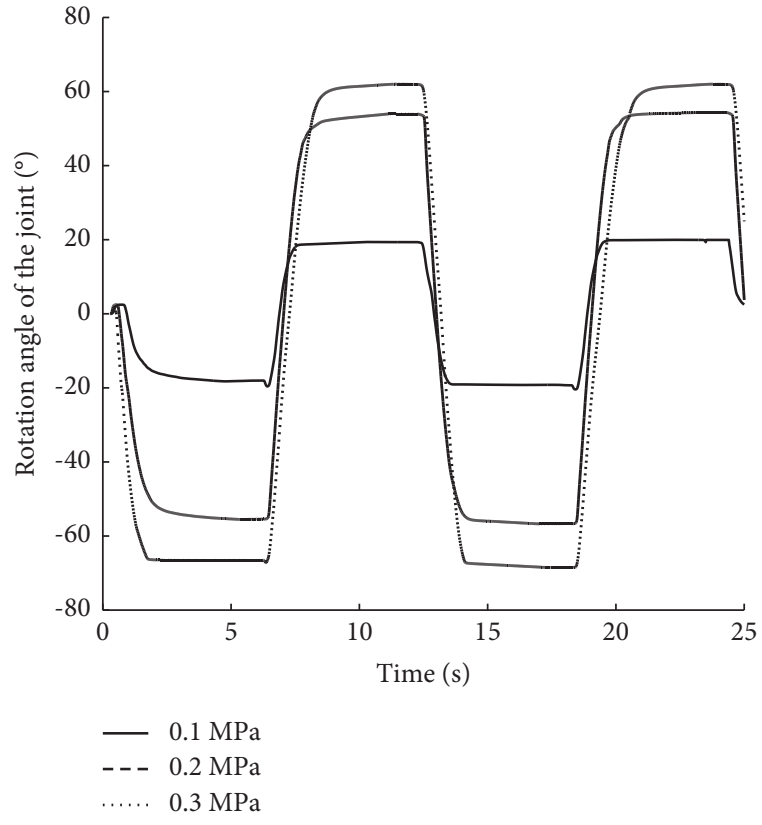

FIGURE 18: Changes in the joint rotation angle with time.

position. Secondly, a step signal excitation of the same air pressure was provided to the clockwise actuator, while its counterclockwise counterpart was relieved. When the joint rotated clockwise and reached the stable angle, the clockwise actuator was relieved, and the counterclockwise actuator was charged. The joint returned to the initial position, completing a step excitation of forward and reverse bidirectional rotations through the two steps shown above. The response curves outlining the relationship between the joint angle and angular velocity under different air pressures were obtained by changing the air pressure step excitation, as shown in Figures 18 and 19. Furthermore, Figure 20 shows the measuring device used in the joint motion experiment.

Figure 18 shows that the joint rotation angle increases with the air pressure. The overshoot is zero when the steady state is reached, which is an overdamping system. The time required for the joint to rotate to the maximum angle of $68.5^{\circ}$ at $0.30 \mathrm{MPa}$ pressure was $0.85 \mathrm{~s}$.

According to the analysis provided in Figure 19, the maximum angular velocity of the joint at the $0.30 \mathrm{MPa}$ pressure was $130 \%$ s. The angular velocity curve fluctuated when the joint reached the maximum angular velocity, mostly since the joint fluctuated slightly after reaching the maximum angle. Additionally, the joint rotated in the reverse direction under the pressure step signal of $0.10 \mathrm{MPa}$. The main reason was that the joint installation preload was $0.12 \mathrm{MPa}$; thus, both actuators were initially in the prestretched state. Finally, the airbag belongs to the hyperelastic body, which can store a certain amount of energy when deformed. On the contrary, the energy released by the working actuator when the pressure was relieved to $0.10 \mathrm{MPa}$ was lower than the energy released by the nonworking actuator when the pressure was relieved to $0 \mathrm{MPa}$, resulting in a transient force imbalance. 


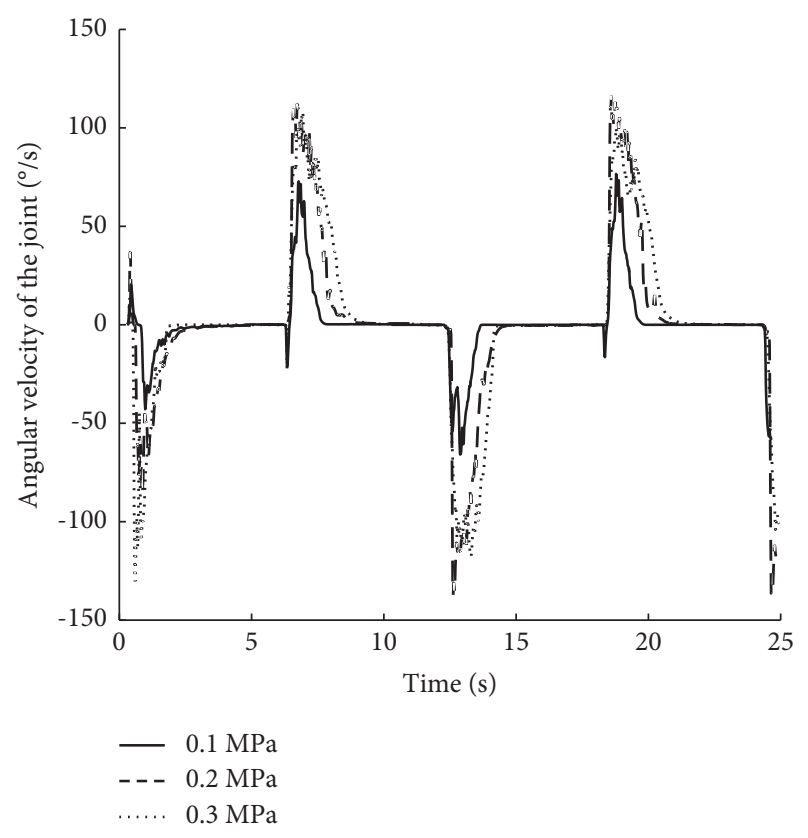

Figure 19: Changes of the joint angular velocity with time.

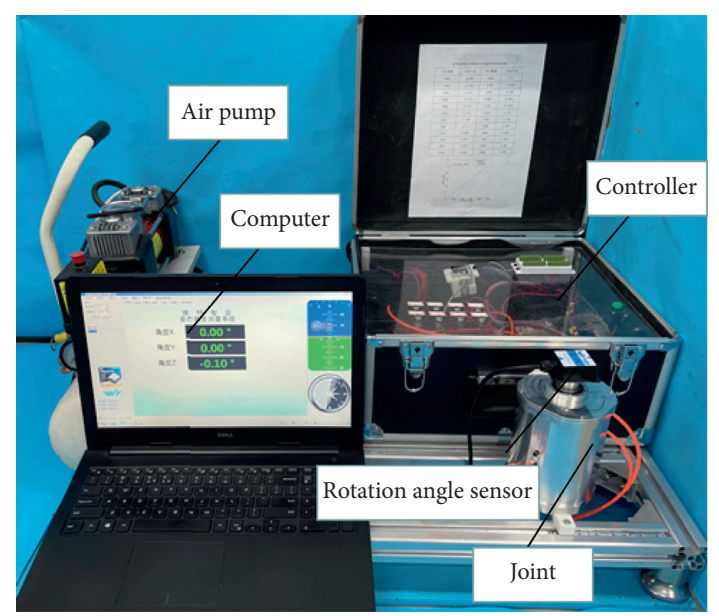

FIgURE 20: Experimental and measuring devices.

\section{Conclusion}

In this paper, a pneumatic bidirectional flexible joint with controllable damping was designed based on the antagonism principle. The proposed joint can accurately adjust the direction of rotation and the driving force size according to the working requirements. The joint structure and working principle were described in detail, and the relevant mathematical model was established. Finally, a series of experiments were carried out, and the following conclusions were drawn based on the results:

(1) The static model of the actuator rotation angle was established, and associated mathematical expressions were provided. Deviations between the experimental data and the theoretical calculation results were analyzed through the actuator rotation angle characteristics. The experimental results have shown that the rotation angle increases nonlinearly with the air pressure. When the air pressure was $0.24 \mathrm{MPa}$, the maximum rotation angle was $142.9^{\circ}$.

(2) The experimental study on the actuator driving capacity (after coming in contact with the object at different rotation positions) has shown that the actuator driving capacity increases approximately linearly with the air pressure. Moreover, the driving capacity was also related to the limit plane when in contact with the object. The underlying rule is that the closer the limit plane is to the actuator rotation starting point, the higher its driving capacity will be. Therefore, the actuator had the highest driving capacity near limit plane 1 . The maximum actuator driving torque reached $4.76 \mathrm{Nm}$ for the air pressure of $0.24 \mathrm{MPa}$.

(3) A pneumatic ring brake was designed, and the relevant theoretical model was established and verified experimentally. The experimental results have shown that the braking torque increases approximately linearly with the air pressure. When the air pressure was $0.32 \mathrm{MPa}$, the maximum braking torque was $4.21 \mathrm{Nm}$.

(4) The joint antagonism experiment was carried out, and the relation between the rotation angle and the driving torque with the air pressure was obtained. The optimal joint preload obtained through the symmetrical pressure experiment was $0.12 \mathrm{MPa}$. On this basis, the motion characteristics of joint angle and angular velocity experiments were completed. The experimental results have shown that the rotation direction and the joint driving force can be accurately adjusted by controlling the actuator air pressure. For the excitation of the step signal, the joint response time was $0.85 \mathrm{~s}$ when the joint rotated to the maximum angle of $68.5^{\circ}$ at $0.30 \mathrm{MPa}$.

This new flexible joint has several advantages, including the large driving and braking forces, low cost, and easy to fabricate and repair. It is expected that the proposed design will be used in applications such as the service industry, rehabilitation robots, and agriculture harvesting. In the future, the authors aim to further optimize the actuator structure, primarily to achieve the joint rotation angles above $90^{\circ}$ in both forward and reverse directions. At the same time, the joint weight should be reduced to reduce its influence on performance.

\section{Data Availability}

The data used to support the findings of this study are available from the first author (corresponding author) upon request.

\section{Conflicts of Interest}

The authors declare that they have no conflicts of interest. 


\section{Acknowledgments}

The authors would like to thank the Natural Science Foundation of China (NSFC) (nos. 51275004, 51206011, and U1937201), Jilin Province Science and Technology Development Program (nos. 20200301040RQ and 20180201050GX), and Project of Education Department of Jilin Province (nos. JJKH20190541KJ and JJKH20190640KJ).

\section{References}

[1] X. Y. Fu, W. Wang, L. Wang, H. Wei, and C. Yun, "Analysis and study on dynamical characteristics of variable stiffness joint actuator," Robot, vol. 39, pp. 466-473, 2017.

[2] R. F. Li and G. Q. Ma, "Dual-arm kinematics characteristics analysis of humanoid robot based on matlab," Journal of Huazhong University of Science and Technology (Natural Science Edition), vol. 41, pp. 343-347, 2013.

[3] Z. M. Zhang, Z. Z. Chen, and Y. J. Gong, "The design and angle characteristic experimental of plane confrontation joint with pneumatic artificial muscle," Hydraulics Pneumatics \& Seals, vol. 35, pp. 36-39, 2015.

[4] J. F. Li, Z. K. Zhang, and L. Y. Dong, "Development of parallel ankle rehabilitation robot and motion control strategy," Journal of Central South University, vol. 50, pp. 2753-2762, 2019.

[5] D. K. Liang, Y. H. Chen, and N. Sun, "Overview of control methods for pneumatic artificial muscle-actuated robots," Control and Decision, vol. 36, pp. 27-41, 2021.

[6] J. Zhu, C. Y. Huang, J. T. Lei, and B. Qi, "Position/stiffness control of antagonistic bionic joint driven by pneumatic muscles actuators," Journal of Mechanical Engineering, vol. 53, no. 13, pp. 64-74, 2017.

[7] V. Oguntosin and A. Akindele, "Design and characterization of artificial muscles from wedge-like pneumatic soft modules," Sensors and Actuators A: Physical, vol. 297, Article ID 111523, 2019.

[8] V. Oguntosin, W. S. Harwin, S. Kawamura, J. N. Slawomir, and H. Yoshikats, "Development of a wearable assistive soft robotic device for elbow rehabilitation," in Proceedings of the 2015 IEEE International Conference on Rehabilitation Robotics (ICORR), pp. 747-752, Singapore, August 2015.

[9] J. Fras, Y. Noh, H. Wurdemann, and K. Althoefer, "Soft fluidic rotary actuator with improved actuation properties," in Proceedings of the 2017 IEEE/RSJ International Conference on Intelligent Robots and Systems (IROS), pp. 5610-5615, Vancouver, BC, Canada, September 2017.

[10] V. Oguntosin and A. Abdulkareem, "Hand gesture control and design of a rotary pneumatic soft actuator using leap motion sensor," International Journal of Intelligent Robotics and Applications, vol. 4, no. 3, pp. 328-341, 2020.

[11] A. Ainla, M. S. Verma, D. Yang, and G. M. Whitesides, "Soft, rotating pneumatic actuator," Soft Robotics, vol. 4, no. 3, pp. 297-304, 2017.

[12] J. Yan, B. Xu, X. Zhang, and J. Zhao, "Design and test of a new spiral driven pure torsional soft actuator," in Proceedings of the 10th International Conference on Intelligent Robotics and Applications (ICIRA), pp. 127-139, Wuhan, China, August 2017.

[13] J. Yan, X. Zhang, B. Xu, and J. Zhao, "A new spiral-type inflatable pure torsional soft actuator," Soft Robotics, vol. 5, no. 5, pp. 527-540, 2018.

[14] X. M. Liu, Y. W. Zhao, D. X. Geng, C. Shoue, T. Xiaobo, and C. Changyong, "Soft humanoid hands with large grasping force enabled by flexible hybrid pneumatic actuators," Soft Robotics, vol. 8, pp. 175-185, 2020.

[15] M. Manti, V. Cacucciolo, and M. Cianchetti, "Stiffening in soft robotics: a review of the state of the art," IEEE Robotics and Automation Magazine, vol. 23, no. 3, pp. 93-106, 2016.

[16] Y. Yang, Y. Li, and Y. Chen, "Principles and methods for stiffness modulation in soft robot design and development," Bio-Design and Manufacturing, vol. 1, no. 1, pp. 14-25, 2018.

[17] C. Laschi, M. Cianchetti, B. Mazzolai, L. Margheri, M. Follador, and P. Dario, "Soft robot arm inspired by the octopus," Advanced Robotics, vol. 26, no. 7, pp. 709-727, 2012.

[18] F. L. Han, P. Li, M. H. Li, and T. Liang, "Design and motion simulation of variable stiffness bending soft actuators driven by granular flow," China Mechanical Engineering, vol. 31, no. 8, pp. 890-897, 2020.

[19] T. Hou, X. Yang, Y. Aiyama et al., "Design and experiment of a universal two-fingered hand with soft fingertips based on jamming effect," Mechanism and Machine Theory, vol. 133, pp. 706-719, 2019.

[20] Y. Wei, Y. Chen, T. Ren et al., "A novel, variable stiffness robotic gripper based on integrated soft actuating and particle jamming," Soft Robotics, vol. 3, no. 3, pp. 134-143, 2016.

[21] L. Yancheng, L. Jianchun, T. Tongfei, and L. Weihua, "A highly adjustable magnetorheological elastomer base isolator for applications of real-time adaptive control," Smart Materials and Structures, vol. 22, Article ID 095020, 2013.

[22] F. X. Wang, Z. G. Xing, P. P. Yang, J. Lin, and J. Zhao, "Study on steady-state heat transfer of soft manipulator with variable stiffness based on low-melting-point," China Mechanical Engineeringineering, vol. 32, pp. 793-798, 2021.

[23] R. Zhao, Y. Yao, and Y. Luo, "Development of a variable stiffness over tube based on low-melting-point-alloy for endoscopic surgery," Journal of Medical Devices, vol. 10, 2016.

[24] F. Y. Xu, Q. S. Jiang, F. Y. Jiang, J. Shen, X. Wang, and G. Jniag, "Design and testing of a soft robot with variable stiffness based on jamming principles," Journal of Mechanical Engineeringineering, vol. 56, pp. 67-77, 2020.

[25] M. E. Giannaccini, C. Xiang, A. Atyabi, T. Theo, N.-M. Samia, and D. Steve, "Novel design of a soft lightweight pneumatic continuum robot arm with decoupled variable stiffness and positioning," Soft Robotics, vol. 5, pp. 54-70, 2017.

[26] B. Mazzolai, L. Margheri, P. Dario, and L. Cecilia, "Measurements of octopus arm elongation: evidence of differences by body size and gender," Journal of Experimental Marine Biology and Ecology, vol. 447, pp. 160-164, 2013. 